tron, when the diceace ogain showed itecif, and he was sudnitted, Oot 9th. He was put at once on the treatmant lact mentioned, and up to the present dato bo is rectity mending.

Fite may other diecases of the ekin, this appeared at the epring of the year, and again at the fall. Is the discame of the secondary or tertiary sequelse of syphilis, or is it an example of hereditary syphilis, or is it "scrofuloas"? Many instances of secondary syphilitic ulceration sboat the als of the nose and lips have fallen under me notice, which have immediately yielded to the protoiotide of mercury combined with quinine and opium. I partioularly bear in mind one case of a female patient at the Western Dispensary, Westminster, who, I see, by reference to my case-book, came under my care for the first time in the year 1848. She suffered from ulceration on the side of the nowe and edge of the lower lip. She had syphilis commanicated to her by her husband twenty years previously acounding to her own account. She was treated successfully with the proto-iodide. I find she was again under my twe tment in 1851; and again the same medicine overcame the disease, though iodide of potassium failed to do so. At the same Dispensary, about two years since, a man was sent to mo with a frightful ulcer cutting half through the lower lip; it was supposed to be a case of cancer, and certainly had many of the characteristics of that disease. I treated it for syphilis by the proto-iodide of mercury, and healed the sore. My diagnosis was confirmed by the fact of the wife having some small ulcers, about the nature of which there could be no doubt, on the outer side of the knee.

Of the influence of very minute doses of bichloride in healing unhealthy sores, I have also, as in the case of the porter above detailed, seen marked examples.

I gave to a little girl, at. 4 years, who suffered from large ulcerations of the integument over exostoses, the sicticth of a grain of the bichloride with the best result, after I had exhausted every other means. I think we are justified in suspecting that very small doses of the bichloride of mercury will often act as a powerful tonic.

Iondon, October 1855.

\section{origntat COMMUNICATIONS.}

\section{S ON BRIGHT'S DISEASE. \\ By ROBT. C. R. JORDAN, M.B., etc. \\ [Read before the Medical Society of Queen's College, Birmingham.] \\ [Concluded from page 056.]}

THe chronic form of albuminous nephritis can sometimes be traced as a direct sequence of the acute; but in many cases, where we think we have such a history, there is a fallacy, from the fact that the acute disease very often supervenes on the chronic. No doubt there is always a congestive stage; but this is so gradual that it is rarely discovered by the patient, and is only seen in those cases where the medical attendant almost expects it. Dr. G. Johnson has well described the signs noticeable in the urine in the early stages of the disease. He says, "The case in which the search is most likely to be successful is in chronic gout, and especially when concretions are beginning to form in the joints." The urine chould be examined in these cases when "the gouty paroxym is subsiding, and it will have the following characters. In quantity, specific gravity, and colour, it will differ very little, if at all, from the standard of health; it is perhape more acid than usual, but without a trace of albuwen." After standing, a codiment falls, which, on exmination, is found to consist of cylinders or caets from the tabes: 80 that desquamation has begun to take place; in .other words, either owing to, or accompasied with,

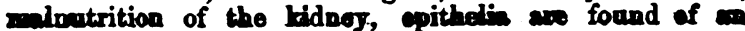

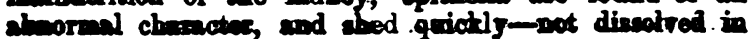

the couretion, as is probable in the ordinary hoalthy state, but disintegrated, ihrink up, and withered. It of importance to detect the diseres thus early, becanes in this stage it is more amenable to treatinent. With each succeeding attack of gout, the epithelial elements are shed more abundantly, until albumen appears in the urine, the congestion then having reached the point at which nature relieves itself by the escape of the serum. Probably this was the case before, only the proportion of albormen was too small to be appreciable by chemical tests.

But it is more often, instead of thus tracing the onset of the disease, that we find the urine of patients to be albuminous who have never themselves suspected they were the subjects of renal disease; and the following account expresses the usual series of symptoms related to the medical attendant.

“ He has latterly been growing pale, and his strength seems to have failed. In addition to a general feeling of lassitude, he has been inclined to be sick in the morning, and occasionally he has noticed his feet and hands to be swollen, and his face slightly puffy; the skin has been dry, and there has been more or less pain in the back, which bo has referred to weakness. The urine used to be very copious, of a pale colour, and passed frequently; he was obliged to get up at night to pass it; latterly, though amall in quantity, he still has to pass it often, and has usually to get up in the night for this purpose."

Such is the common history of the state of the patient before applying for relief. Perhaps the first discovery of the disease may be the supervention of an acute or subacute attack upon the chronic. He "catches a fresh cold"; there is consequent increase of kidney disorder; and a few days' illness, with slight dropsy and scanty secretion of urine, may end fatally, either by coma, or inflammation of one of the serous membranes, more usually the pericardium. But here I am speaking only from memory, not by numerical computation; and, in the table constructed by Frerichs, peritonitis is recorded as the most frequent.

In these sudden and highly dangerous attacks, activo measures must be resorted to. Elaterium must be given internally, and the hot air-bath used night and morning. If there be a tendency to coma, blisters may be applied to the nape of the neck; and if inflammation of the serous membrane, pericardium, or pleura, be present, blisters may be applied in their vicinity. If the patient be not very weak and anæmic, a few leeches may be used. I am aware that in thus advocating blisters there is said to be danger from the effects of the cantharides on the kidneys; but if the skin be protected from the immediate contact of the plaster by oiled paper, this will be very rare: nor, I think, is the danger sufficient to deter us from using a means undoubtedly often of the greatest benefit. I have often used blisters with much good, and never seen any ill effects from them. The drain of serum from the vessels, as well as the counterirritation, is of use in these cases.

The patient may return from this attack to his previous chronic condition, or he may die in it, or the recovery may be incomplete. The general result is, that each of these fresh accessions considerably increases the mischief in the kidneys. He thus gets gradually worse; dropsy, in the shape of general cedema of the areolar tissue, comes on, which yields now to no remedies; or perhaps he may rocover from one or two attacks of chronic dropsy, but another time it may again return, and not improve by treatment. He gradually sinks worn out in this manner, much harassed by the dyspnoen from effusion into the pulmonary tissue. The secretion of urine is much diminished towands the latter stages of the disease, and the quantity of abumen in the urine lessened. This is explained by the atrophic condition of the kidney. Effusion takes place into the serous cavities also; and death takes place, partly from exhaustion, partly from the mechenical effects of tho dropsy, generally acoompenied towards the end by more or leas of coms, perhaps from cedema and consequent pressurs on the brain. Such seems to me to be the natural terming 
tion of the disease, though many writers regard a mode of denth more purely by coma in this light. But it is rarely that the disease is allowed to come to this termination. There are many accompaniments to it in the shape of other diseases, which generally cause the death of the patient before he is thus completely exhausted.

Among the most general accompaniments to this chronic form is gout, in its varied forms; and in these cases, it may be here stated parenthetically, we should be very careful about the use of colchicum, except in very minute quantities; and indeed it must be remembered generally in treating these diseases, that much smaller doses should be given than at ordinary times; probably because the secreting power of the kidney is impaired, and consequently less eliminated as waste from this source. Gout is so frequent an accompaniment that the chronic stages of this malady are termed by Dr. Todd gouty kidney. The well known absence of gout in Edinburgh may account for some of the modifications which the disease certainly there undergoes.

Diarrhoea is thus stated by Dr. Christison to be the most frequent termination, and is indeed so at the present time in the Edinburgh wards; yet this is comparatively zare in London, and generally purgatives have to be given. It is, however, a termination which sometimes occurs, requiring care in its checking. One of the most efficient astringents in these cases is the decoction of logwood; it howerer acts on the kidney also, but never injuriously in the cases in which I have seen it administered. The urine is coloured by it, and this decolouration must be distinguished from blood carefully, either by the microscope or by nitric acid, which will render the colour of the log wood urine much more brilliant, but will only coagulate the albumen in that containing blood.

A more common malady in England is inflammation of one or more of the serous membranes, and of the pericardium most frequently. Death often results from this. Mercury may sometimes be used in these cases with advantage, though it is to be given with caution. In kidney disease, it is not wholly to be rejected, and I have seen good result from it; but very small doses sometimes salivate. Blisters also should be applied over the heart, but effusion and death are frequent in this complication.

Carbuncles, as in diabetes, are sometimes an accompaniment of this disease, and I have seen them materially assist in its downward course.

Vomiting is often a troublesome symptom, sometimes eren a dangerous accompaniment. Minute doses of hydrocyanic acid here have the best effect.

Coma may often terminate life; and this may spring from two causes: 1. Edema of the brain, generally then accompa. nied with dropsy of the areolar tissue in the whole body, and of the lungs also ; so that it is impossible to say how far the impeded respiration may be the source of the coma-how far the œdema, and consequent pressure on the brain : and 2. Suppression of urine. In $\mathrm{my}$ experience, a dry state of brain, with coma, is not so frequent as an odematous condition, but it is not yet very rare; and Cases XvI and XvIr in Dr. Johnson's work may be quoted as examples. It is no use here theorising as to whether urea or carbonate of ammonia be the cause, or some other morbid constituent; suffice it to state the known fact that suppression and coma are almost always constant companions. These states of coma are difficult to treat. Leeches to the head and sinapisms to the feet are useless in the latter stages, though in the earlier they may give more relief. In my opinion, more good results from blisters and the drain of serum than any cther remedies. The hot air-bath must be resorted to; even diuretics in some cases (of these, however, we shall speak again); and certainly purgatives as elaterium. Epileptic fits often accompany the early head symptoms; and there is always danger of the patient never recovering from the subsequent coma.

An stheromatous condition of the arteries is sometimes en accompaniment, but not more than in other states of inpaired wutrition. Apoplexy conequent on this is accontingly wometimes a termination.
Endocarditis is a frequent result of this form of diseace, bat in no case that I hare seen has the approseh been anything but gradual and unnoticod; so that it mas rather be termed valvular disease of the heart than ondocarditic The mitral valies are in my experience the most of then affected. This complication of course hastens death. I have never traced it as a consequence; and it has been asserted by many to be a cause, not a result.

From atheroma of the arteries, the aortic ralves are 200 more or less affected.

Pulmonary apoplexy is sometimes result of these cardiac affections; but a more frequent source of danger is pneumonia, consequent on cedema, or bronchitis, from the same cause. The cedema of the lungs is also in itself dangerous. Little can be done in these cases. Docoction of senega may be giren as an expectorant in the bronchitis or adema, in conjunction with cream of tartar, rendered soluble by borax; and blisters may be used.

The use of diuretics in the different stages of this disease is a subject which must not pass without notice; and the clearest summary of what we can say of their action is given in a few words by Dr. Watson. "If we fail to excite the action of the skin, and if diarrheer prevents the use of purgatives, the effusion still continuing, we must choose the least of two evils, and endeavour to remove the dropsy by diuretics, whether these accelerate the progress of the disease in the kidney or not." They are given therefore as a necessary evil; the best and least objectionable seems to be digitalis; and this may be combined with small doses of the soluble cream of tartar. Frerichs says that diuretic are sometimes necessary to wash out the fibrinous concretions in the tubes: he, however, strongly deprecates the general use of these medicines for washing out these coagula; but pure water would be quite as efficacious as any other form of diuretic.

Decidedly, however, the elimination of the fluid should be made by the skin and the mucous membrane of the intestines, if this be at all possible. Diuretics are only a lact resource, when these fail. If the fluid accumbalates in the areolar tissue under the skin, it is sometimes necessary to use surgical means to aroid its bursting of itself. I mach prefer acupunctures (not too near each other) to imcisions. These last do not heal readily, and are apt to slough.

A question of great importance is, Can this chronic form of the disease be cured in the early stages? In the latter, when organic change has taken place in the kidneys, it is of course impossible; but, in the early stages, does it admit of arrest and cure? I am disposed, with Dr. Johnson, to think that this may be the case. I remember well one example which can be quoted of, as it seems to me, perfect recovery. The subject was a young, pallid boy, aged about 12 years, living in a country village. He was brought to me for general lassitude, weakness, and frequent headsche. He was pale, with a dry skin; the bowels were confined; there was loss of appetite, and he was very nervous; at night he was dreamy and restless. On inquiring further, he had not been well since a slight attack of searlatim, about a year before. This led me to the examination of the urine, which was albuminous, and with the character of this albuminous nephritis in its early stages. I treated him for about a month with light tonies, nutritious diet, purgatives, strict regulations as to the avoidance of cold and damp, and, though not preventing out-door exercise, yet limiting it considerably, and enjoining a reclining position when in the house. His general health certainly improved, and the symptoms were alleviated; but the albumen dia not diminish. Under these circumstances, though apparently better, I could not help giving an unfarourable prognosis. He then took as an alterative small doses of iodide of potassium; and under these he improved docidedt?, and the albumen at length disappeared entirely, and and not return.

I have seen now other cares benefited by the same modicine. Its nse is to me empiric only ; certainily it is firurotic, but, at the same time, it increases the action of overy wo creting gland in the system. In another case like the one 
anged I homld be tampted to the the iodide of iron, or

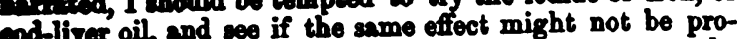
dnoed by them. The hygionic remedies, it must ever be remembered, are of the strongest importance in these cases. The recumbent position should be kept up as far as posible without deteriorating the hesith of the patient by ant of exercise. Of steel tonics, which are generally admissible, the tincture of the muriate is often the best. The digeetive system must be attended to, and the bowels kept not only regular, but with a tendency to be loose. The general plan of treatment should be tonic, but decidedly not atimulant. If the patient be in a condition to render this possible, a sea royage, or change to a warmer climate, may be advisable. The disease is, however, usually only delayed; and, in spite of all treatment, the patient will generally get worse, though the illness may remain for jears before any decided symptoms appear; these have been described previously.

Now, what is the essential character of this disease? The chief deposit of fresh substance seems to be intertubular in the proper matrix of the kidney; the effects on the blood-ressels, and the consequent escape of the albumen and fibrin into the tubes seems to me, aftor the first congestive stage, to be, in a great measure, mechanical. That the inflammatory attack also spreads to the tubes themcelves is, however, proved by the great shedding of epithelial particles which usually takes place. These are either passed out by themselves, or else escape entangled in the fibrinous casts. In some cases, this shedding of epithelium does not take place, and we then have the non-desquamatire disease of Dr. Johnson. This is generally an acute and fatal form of attack. I quote from his account, never having seen an example of this variety ; casts without epithelial cells ("waxy casts") are frequently mixed with others in chronic cases. As in cirrhosis, the liver is first large, then granular and contracted, from the shrinking of the fibrous matrix; so is the kidney first large from congestion and effusion, and afterwards shrinks, until it becomes smaller than natural, hard, and nodulated.

I shall only notice the post mortem appearances found in the kidney itself, and these present many forms, according to the period of the disease. Supposing death to take place in the early stage, the kidney is large, soft, and very rascular, the cones are large, and the cortical substance is congested. The kidney is of a dark chocolate red. The capsule is generally easily stripped from the surface. In less severe cases, the congestion is only partial, and this gives rise to a stellate appearance of vessels on the surface; it may have the appearance of combined anæmia and increased vascularity, whilst it is yet smooth and not distinctly granular. Soon, however, in an uncomplicated case, it loses its smoothness of outline, and assumes the granular form, which earned for it the synonym given to the disease by Christison, of "granular degeneration." The kidney is generally congested in patches, the surface is studded with minute granules, of a light colour, which are more marked almost on section than on the surface. They are formed from a deposit taking place in the dilated portion of the tubes around the Malpighian capillaries. The vessels are here naked, according to Mr. Bowman's view of their anatomy. The deposit scems to take place in these dilated extremities, and also in the tubes, as well as in the cortical substance. The cones are now less vascular, sometimes contracted in the centre, so as to put on a wheatsheaf appearance. In this stage, renal cysts are often met with. As the disease progresses, the kidney becomes more atrophied, the newly deposited substances shrinks more, until, at length, it not only becomes granulated on the surface, but divided into large nodular portions, between which fibrous bands can be seen by the naked eye very plainly. The prevailing colour is in this stage greyish red. Generally, both kidneys are found nearly in the same condition. The capsule in this last stage is thickened, and more adherent, and cysts of variable size are now frequently formed on the surface of the kidney. On section, the cortical substance has undergone the greatest share of the atrophy ; as Dr. Bright expressed it, the cones seem an if drann towands the surface of the kidney.

We have mentioned the renal cysts more than once in this account of the post mortem appearances. What is their origin?

There are, as is well known, three modes by which cystic growths originate, and the cysts have been referred to each of these in turn:-lst. Patches of fluid in the areolar tissue become shut off by inflammation, and thus, isolated from surrounding parts, grow into cysts. Vogel refers these to this origin. 2ndly. Dilatations of the ducts of glands are a well known cause of cysts. Many, amongst whom are Johnson, Gairdner, and Frerichs, refer these kidney cysts to this origin, regarding them as dilated uriniferous tubes to this origin, regarding them the growth of special cells. One of the epithelial cells of the gland takes an abnormal development. Simon originated this view with regard to the cysts of the kidney, and this has since been adrocated by Rokitansky and Paget. Perhaps both of the two latter theories may be at times correct.

Microscopic examination of the kidney brings into view casts such as have occurred previously in the urine, and also casts of a larger character from those tubes which though denuded of epithelium, have not yet shrank and atrophied. There is also much loose epithelium; but as this peels off after death, it may be partly a post mortem result.

There is a peculiar modified form of this inflammatory disease depending on the strumous nature of the subject. It forms the lardaceous kiduey of Rokitansky, the waxy kidney of Johnson. It has been usually found accompanied with the same disease in the liver which has been described by Dr. Budd as scrofulous degeneration. Like other scrofulous inflammations, the product is of an albuother scrofulous inflammations, the in which the liver was analysed, out of twenty solid parts, sixteen were albumen. analysed, out of twenty solid parts, sixte character as the last, The disease seems so be of the same character as the last, but the nature of the inflammatory product modified by scrofula. The mechanical effects are the same, and the patient, and the presence of enlargement of the liver, propatient, and the presence of enlarill help to distinguish it during life. The treatment in such a case offers little hope, but that usually resorted to in scrofulous disease probably benefits the patient most.

Fatty degeneration of the kidneys, which by many is looked upon as a synonym of Bright's disease, is, I believe, very rare. Fatty kidneys are common;* but by fatty degeneration I mean those cases in which the excess of oil is confined to the epithelia in the tubes, and the effects of the disease are wholly mechanical. That such cases may exist, is borne out by analogy, and is prebable; but the generality of cases of fatty kidney are the chronic inflammation before described, only where fatty matter, apparently cholesterine, is deposited, either simultaneously with this adventitious product, or else as a process of degeneration in it. That such is the case, is quickly known by the presence of oil-globules in the epithelia of the casts. It always, when present in the kidney, appears in these cells, perhaps by an effort of nature to remove the morbid product, perhaps because they participate in the degeneration. The symptoms in no way differ from those of Bright's disease generally; but the appearance of the kidney after death is much modified by the presence of the new product. It is much enlarged, smooth in texture, sometimes granular and very pale in colour, both externally and on section; and there are fat granulations scattered through the cortical substance.

There is no difference in treatment or symptoms between this and the more usual form of the disease.

- I would bere wish to direct attention to what seems rather a neglected fact in pathology, that there are two exactly analogous forms of fatty liverone, in which the gland substance is primarly affected; the other, where the deposit of adipose matter superrenes on cirrhosis. Contrary to what 1 be lieve to be the case in the kidney, the second afiection is much the rarces of the tro in the liver. The different appearances which the gland presents in the two caces almoet afford a contragt to oach other. This obeorvation is s. 
Snch is brief sccount of the alterations in the kidney sem the common name of Bright's disease. It has been difficult to bring so wide a subject into so short a space; and this will, it is hoped, account for some of the imperfections of the paper.

Birmingham, October 1855.

\section{CASE OF POISONING BY THE VAPOUR OF COKE: WITH REMARKS.}

By W. F. WADE, M.B., Resident Physician to the General Hospital, Birmingham.

[Read before the Birmingham and Midland Counties Branch.]

Thos. Stephens, a well developed lad, aged 13, was admitted into the General Hospital, Birmingham, under Dr. Fletcher, on Sunday, 25th March, 1855, at 2 o'clock, P.M., under the following circumstances. About an hour previous to his admission, he had been discovered in a sunall room in Floodgate Street, in bed, between his father and an elder brother aged 20 , both of whom were dead. The size of the room ras $430 \frac{1}{t}$ cubic feet: it contained a small stove, in which were the remains of a coke fire, quite extinguished. There was a hole in the wall, the size of one brick, communicating with a chimney of notoriously bad draught. I have not been able to ascertain whether the chimney of the stove had been properly fitted to this hole ; but it is very probable that it had not, as the stove was usually taken out of the room in the daytime. The boy, when discovered, was apparently in a quiet sleep, as were also his father and brother. He was removed into a neighbouring house, where he was immediately attended by Mr. Badger, of Bromsgrove Street, to whose prompt attention he is in a great measure indebted for his recovery. When first seen by Mr. Badger, he was (as I am informed by that gentleman) apparently asleep, with a moderately good pulse, and no appearance of congestion of the face or lips. Mr. Badger immediately dashed him with cold water, upon which he opened his eyes and stared about him, so that every one around thought that he would presently speak. Ile swallowed some gruel, a little brandy, and aromatic spirits of ammonia: some of these he vomited again. Instead, however, of becoming more conscious, he became again quite insensible, and was brought to the hospital, in somewhat less than an hour after his discovery.

On admission, he appeared almost inanimate; the face pale and cold; lips of a pale pink colour, their edges in two or three places slightly excoriated; limbs flaccid; pulse imperceptible, save an occasional feeble wave; heart's action scarcely perceptible; respiration quiet and unembarrassed, but very shallow; no stertor; lower part of chest much dilated, as in emphysema of the lung; the middle intercostal and suprasternal spaces drawn in during respiration; belly much distended hy tympanitis, and hard; pupils normal, perhaps rather contracted; eyes turned upward and inward, as in sleep. If left to himself, his respirations appeared to become shallower and shallower, as if about to cease completely. Dashing him on the face and chest with cold water roused his respiratory powers; but the effect was transient, and the process had to be frequently repeated. His pulse evidently increased in force and constancy when his breathing was more vigorous. Pressing forcibly and suddenly on the epigastrium induced inspiration; but as he was sick directly after one of these operations, I desisted from them; and, on making an effort to open his mouth, to rake the egesta out of his fauces, I found that his jaws were firmly fixed: I, however, forced them open, and got out some food which was lodged on the back of the tongue, so seriously impeding his breathing that, if it had not been promptly removed, it would probably have proved fatal. In the very act of vomiting, his pupils became widely dilated, and remained so afterwards. When he had been in the hospital for abont a quarter of an hour, I gave him a drachm of sulphuric æther, as an injection ser rec- tum : it did not seem to produce any other efiect than that of remoring the tympanitis, which it did completely and permanently. He was also cupped to a small amount over permanently. chest, without producing any obvious result. About an hour after his admission, I placed his feet and legs in mustard and water; this produced intense rigidity of the whole body, and his feet and legs became much redder and decidedly warmer afterwards. This rigidity redder and decidedly for several hours, alternating with persiste convulsions. The indication for treatment ras evidently to maintain his respiration till the poison should be eliminated: this was best done by constantly pinching him, and occasionally holding strong ammonis pinching him, and about three hours after admission, I applied large blister to his back, having previously administered an injection of three ounces of wine and four ounces of beef-tea: this latter was again repeated about nine o'clock, P.M. About half-past nine (i.e., seven and a half hours after admission), he uttered a slight groan; and on being asked, in a loud voice, "where it hurt him", he said, "My back". This was in allusion to the blister which was then rising. It was, howerer, still necessary to keep was then from time to time, as there was a tendency to deep sleep, bordering on coma, during which the respirations became shallow and slow; and it was not till half-past five the next morning that $I$ thought it safe to leave him. At nine the next morning, he was sensible, though still dull and heary; his tongue was covered with a thick white fur, except at the tip and edges, which were red and dry, with enlarged papillæ. During the night, he occasionally asked for drink, and appeared thirsty, but vomited everything except cold water. He passed water in bed about two hours after his admission, and again twice during the night. The consecutive fever increased during the second day; and on the third day his urine was albuminous, and in the evening bloody. On the fourth day, the albumen and fever had nearly disappeared, under the little or no recollection of the events which occurred on the day previous to the occurrence, being totally oblivious of the fact of his having received his wages on that (Saturday) afternoon.

Rexakks. Poisoning by the vapour of burning coke has not, so far as I am aware, been hitherto noticed ;* but from the fact that good coke is approximately pure carbon, prepared from coal instead of from wood, as well as from the history of the foregoing case, there is no reason to believe that any material difference exists between the symptoms of poisoning by coal charcoal and wood charcoal vapours. Coke, however, not unfrequently contains sulphur, the sulphureous acid gas arising from the combustion of which is an extremely energetic irritant poison, acting especially on the air passages. The gases resulting from the combustion of charcoal are carbonic acid and carbonic oxide (Guerard), carburetted hydrogen (Orfila), and probably a pyrogeneous acid (Hünefeld), whose nature is not accurately determined. These gases vary in relative amount, somewhat in proportion to the vigour of the ignition. As the fire had gone out in the present instance, it is clear that the victims had suffered from the combination of all these poisons.

These gases, though when undiluted they induce death by spasmodic closure of the glottis, and so act as a negative poison by the exclusion of oxygen, yet, when diluted with air, may be readily inspired, and then act as a positive sedative poison. This has been showu in different ways, and by different persons; but by none more distinctly than by Rolando, who, having found that the common land tortoise was not injured by the simple occlusion of one lung, contrived that one of these animals should breathe pure air by one lung, and carbonic acid gas by the other; and in a ferr hours it died.

- The only rase that 1 am aware of is that mentioned by Dr. Bird, in which o man was poisoned by the vapours arising from the burning of mixture of coal ashes with chalk. (Guy's Hospital Reports, No. VIII, April 1889.) 